\title{
GUEST INFLUENCE ON THE DIPOLAR ASSOCIATION OF THE NEMATIC LIQUID CRYSTAL HOST *
}

\begin{abstract}
D. BAUMAN
Institute of Physics, Technical University, ul. Piotrowo 3, 60-965 Poznań, Poland

Zhong-Xiao Fan and W. HaAse

Institute of Physical Chemistry, Technical University, Petersen str. 20, 6100 Darmstadt, Germany

(Received April 22, 1991)

X-ray diffraction method has been used to study the molecular organization in 4-n-pentyl-4'-cyano-p-terphenyl doped with guest dye molecules. It has been found that the dye with strong polar end group affects significantly the monomer to dimer ratio in the nematic phase of the liquid crystal studied.
\end{abstract}

PACS numbers: $61.30 \mathrm{Gd}, 61.10 ., 64.70 . E \mathrm{w}$

\section{Introduction}

The vast majority of nematogens is composed of polar molecules. The part of permanent dipoles in determining nematic liquid crystalline properties has been the subject of considerable discussion. The first theory of the nematic phase, proposed by Born [1], was based on purely dipolar interactions. However, when the compounds were found that do not possess permanent dipole moments, but do nevertheless exhibit the nematic phase (e.g. alkylazobenzenes [2]), Bori's theory was abandoned. In the first successful theory of the nematic phase, given by Maier and Saupe [3], only interactions between induced dipoles have been taken into account. Although the interactions between permanent dipoles do not play

*This work was partially supported by the Polish Grant of Ministry of Education and Poznan Technical University and partially by Deutsche Forschungsgemeinschaft. 
a significant role in the stabilization of mesophase [4], they are important in determining some physical properties of liquid crystals, especially in the case of the strongly polar substances [5].

The major effect of dipolar end groups in mesogenic molecules is observed with the dielectric properties of liquid crystals. The results obtained from dielectric measurements for compounds consisting of molecules with strongly polar cyano-, nitro- or isothiocyanato- terminal groups have been interpreted in terms of anti-parallel dipolar association of molecules [6-13]. Molecular association can also be observed by the study of the X-ray diffraction of liquid crystals, as it has been shown in [14-17].

In our previous papers it has been found that addition of some dyes to the nematic liquid crystals ("guest-host" systems) changes the amount of the associated molecules, what influences the short-range order in the nematics [12, $17,18]$.

Here, we present the results of the X-ray diffraction studies of the dipolar association of 4- $n$-pentyl-4'-cyano- $p$-terphenyl doped with one of azo dye using different guest concentration.

\section{Experimental}

M a t e r i a l s: The purified 4- $n$-pentyl-4'-cyano-p-terphenyl (T15) was supplied by Merck Company, Darmstadt, Germany. The azo dye with the following molecular structure: was synthesized and chromatographically purified in the

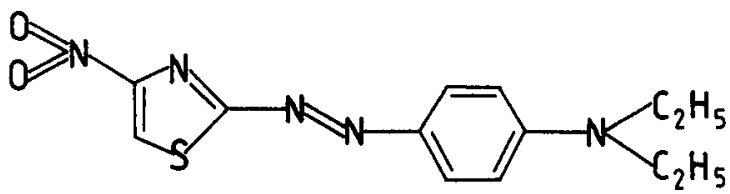

Institute of Dyes, Łódź Technical University, Poland. The concentration of the dye in the nematic host was changed from $3.3 \times 10^{-3} \mathrm{M}$ to $10^{-1} \mathrm{M}$. A p p a r a t u s: The temperatures of the crystal-nematic and nematic-isotropic phase transitions of the dye-T15 mixtures were determined by means of DSC measurements using Du Pont Thermal Analyzer 990 and a polarizing microscope (Leitz Orthoplan-Pol) in conjunction with a Mettler hot-stage FP-82. The experimental errors by melting and clearing points determination are \pm 0.2 and \pm 0.1 , respectively.

The X-ray diffraction measurements were performed with a modified STOE focussing horizontal diffractometer (STOE STADI 2). The X-ray source was a long fine focus X-ray tube SIEMENS FK with a line focus of $0.4 \times 12 \mathrm{~mm}^{2}$. The $\mathrm{CuK}_{\alpha 1}$ radiation $(\lambda=1.54 \AA$ ) was focused using a curved $\mathrm{Ge}(111)$ monochromator. For the fast diffractometry a linear position sensitive detector (STOE Mini PSD) was used with a resolution of the stepwidth $0.01^{\circ}$ in the $2 \theta$ circle. The inner diffraction maxima were recorded at different temperatures in the nematic phase of specimens. The macroscopic orientation of the liquid crystal and dye molecules 
was achieved by means of an external magnetic field of about $0.8 \mathrm{~T}$ using two permanent magnets. The temperature was measured and regulated by using a differential temperature regulator (Thor-PID) with an accuracy of $\pm 0.01 \mathrm{~K}$. Further details of the experimental technique have been given earlier [19].

\section{Results and discussion}

\section{Phase transitions}

It is well known that the addition of a guest dye to a nematic liquid crystal changes the range of the mesophase of the nematic host, causing either a decrease or an increase of the nematic-isotropic transition temperature as well as a decrease of the melting point [17, 20-22]. Moreover, it was found [21] that the presence of the dye in the nematic matrix leads to the formation of the two-phase region in the vicinity of the clearing point. Such a region, in which both nematic and isotropic phases coexist in equilibrium is consistent with thermodynamic laws for binary mixtures and the first-order nature of the nematic-isotropic transition [23]. Since this region is quite narrow for molecules having approximately the same molecular structure, the most workers have failed to observed it previously for elongated dye molecules [20, 24-26].

The phase behaviour of the dye-liquid crystal mixtures studied in this paper at the nematic-isotropic phase transition is illustrated in Fig. 1a. Here $x$ is the molar fraction of the dye and $T^{*}\left(=T^{\prime} / T_{\mathrm{NI}}\right)$ is reduced temperature determined with respect to the clearing point of the pure host, $T_{\mathrm{NI}}=513.2 \mathrm{~K} . T^{\prime \prime}$ is equal to $T_{\mathrm{N}}$ or $T_{\mathrm{I}}$, where $T_{\mathrm{N}}$ and $T_{\mathrm{I}}$ are temperatures at which the first isotropic drop appears and the last nematic drop disappears, respectively, by heating of the guest-host mixture. The two-phase region for azo dye-T15 mixture in the range of the concentration used in our investigation is seen very distinctly.

Fig.1b presents the concentration dependence of the crystal-nematic transition temperature for T15 doped with the dye. A two phase region could not be observed in this case. The reduced temperature, $T_{\mathrm{CN}}^{*}$ is now defined by $T_{\mathrm{CN}}^{*}=$ $T_{\mathrm{CN}}^{*} / T_{\mathrm{CN}}$, where $T_{\mathrm{CN}}$ and $T_{\mathrm{CN}}$ are the melting point of the T15-dye mixture and pure $\mathrm{T} 15$, respectively. $T_{\mathrm{CN}}=404.8 \mathrm{~K}$.

From results presented in Figs.1a and $1 \mathrm{~b}$ it is seen that the azo dye used destabilizes the mesophase of the nematic host, what is in good agreement with the results obtained previously $[13,17,21,27]$.

\section{Inner diffraction maxima}

Typical diffraction patterns for oriented nematics show two main reflections: inner (meridional) reflection related to a molecular length and outer (equatorial) reflection corresponding to a molecular distance [28]. Information about molecular packing is available by measuring the intensity of the diffraction maxima along the meridional direction. Therefore only the inner reflection were studied in this paper. 

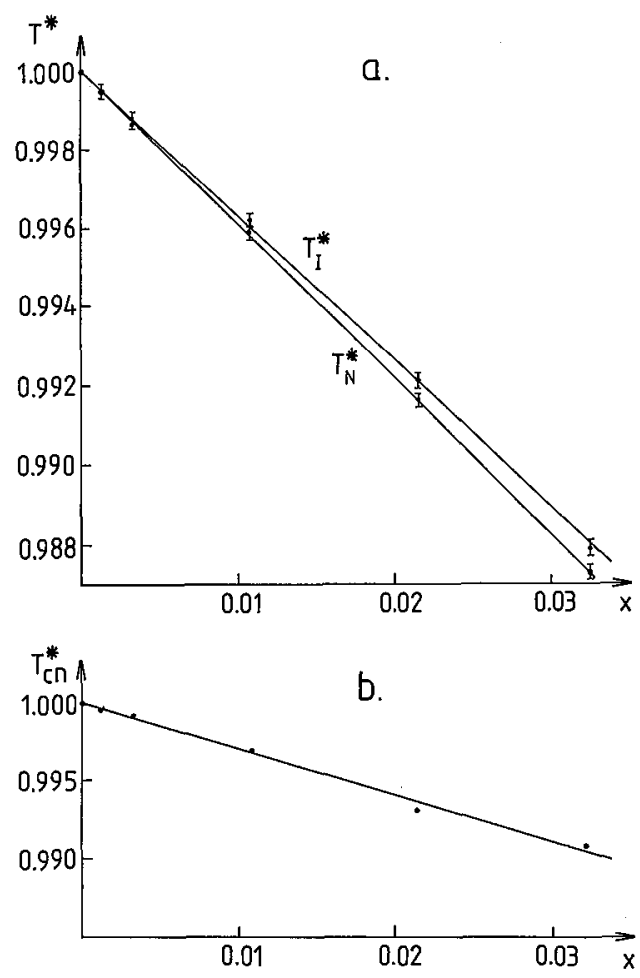

Fig. 1. The reduced temperature $\left(T^{*}\right)$ - guest mole fraction $(x)$ diagram for the azo dye in T15: a) nematic-isotropic transition, b) crystal-nematic transition .

As it has been shown previously [15-17], the inner reflection of T15 consists of two maxima, whose intensities ratio is changed with increasing temperature. The positions of these maxima corresponding to the values of $d_{\mathrm{D}} \cong 31$ Aand $d_{\mathrm{M}} \cong 21$ Åhave been attributed to the dimer (in anti-parallel configuration proposed in [16]) and to the monomer lengths of T15 molecule, respectively $[16,17]$. The addition of the azo dye, used in present studies, does not affect essentially the $d_{\mathrm{M}}$ value of $\mathrm{T} 15$, while $d_{\mathbf{D}}$ decreases somewhat with increasing dye concentration. The presence of this dye changes, however, distinctly the intensities ratio of both inner maxima with respect to that of the pure T15. This is illustrated in Fig. 2, where the intensity profiles of the inner reflection of the pure T15 (Fig. 2a, b), of the mixtures of T15 with the dye at concentrations of $10^{-2} \mathrm{M}$ (Fig. 2c, d) and of $10^{-1} \mathrm{M}$ (Fig. 2e, f) at two various temperatures are presented.

In order to separate the both peaks in inner reflection, the diffraction patterns were fitted to the second power of the Lorentz function. The best fittings are shown in Fig. 2. Then, the total intensities of the separated peaks, $I_{D}(\theta)$ and $I_{\mathrm{M}}(\theta)$, connected with dimers and monomers, respectively, are calculated. The fitting and calculations were made using the STOE-Package computer programs. 


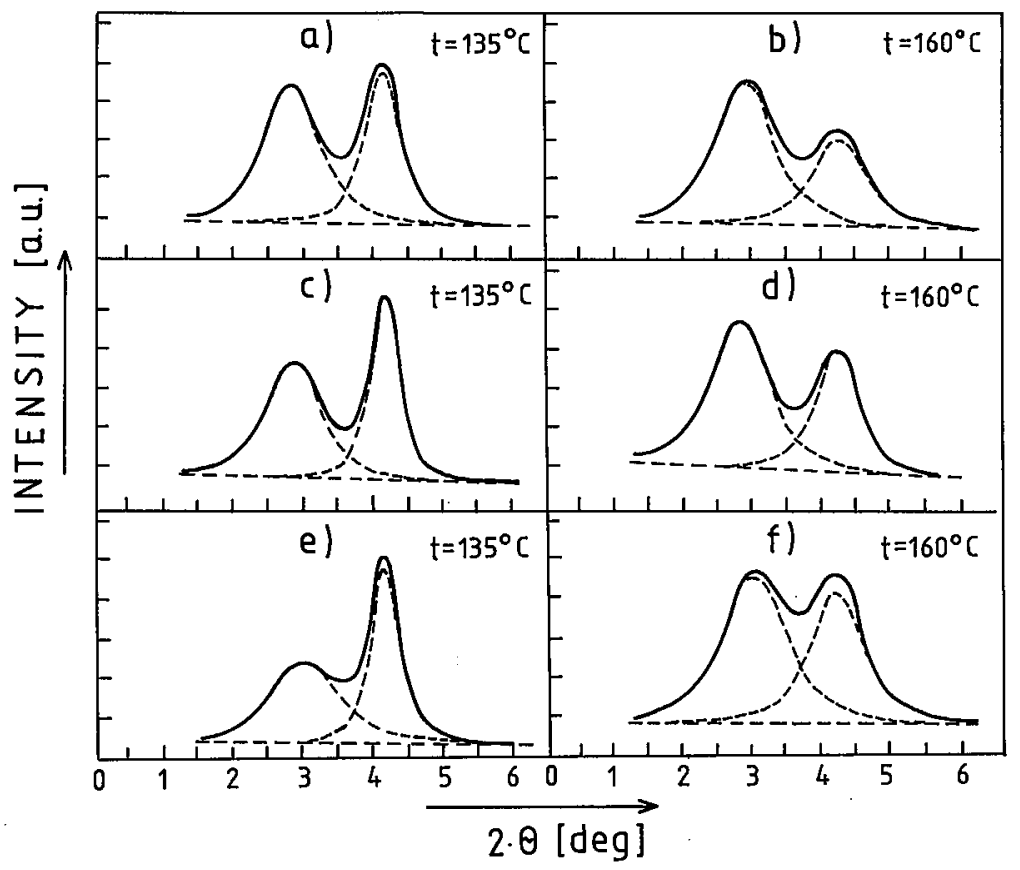

Fig. 2. The intensity profiles of the inner reflection in the X-ray diffraction patterns for the pure T15 (a,b) and the mixtures of the T15 with the azo dye at concentration of $10^{-2} \mathrm{M}(\mathrm{c}, \mathrm{d})$ and of $10^{-1} \mathrm{M}(\mathrm{e}, \mathrm{f})$.

The dimer fraction $x_{D}$ in the specimens investigated can be calculated from the intensities ratio, $I_{\mathrm{D}} / I_{\mathrm{M}}$. Let $n_{\mathrm{M}}$ is the number of monomers and $n_{\mathrm{D}}$ is the number of dimers. Then $n_{\mathrm{D}}+n_{\mathrm{M}}=n$ is the total number of particles. Assuming that $I_{\mathrm{D}} / I_{\mathrm{M}}$ is proportional to $n_{\mathrm{D}} / n_{\mathrm{M}}$, one obtains for the dimer fraction:

$$
x_{\mathrm{D}}=\frac{n_{\mathrm{D}}}{n}=\frac{n_{\mathrm{D}}}{n_{\mathrm{M}}+n_{\mathrm{D}}}=\frac{I_{\mathrm{D}} / I_{\mathrm{M}}}{1+I_{\mathrm{D}} / I_{\mathrm{M}}} .
$$

Fig. 3 presents the temperature dependence of the dimer fraction, $x_{\mathrm{D}}$ measured for the pure T15 and the T15 doped with the azo dye at various concentration, whereas in Fig. 4 the dimer fraction as a function of the dye concentration at reduced temperature $T_{\mathrm{R}}=0.850$ is shown. The reduced temperature, $T_{\mathrm{R}}\left(=T / T_{\mathrm{NI}}^{\prime}\right)$ is now determined with respect to the clearing point of the given specimen $\left(T_{\mathrm{NI}}^{\prime}\right)$ and $T$ is the temperature of the measurement. In the case of the dye-T15 mixture $T_{\mathrm{NI}}^{\prime}$ is the average value of the temperatures at which two-phase region begins and ends.

The results presented in Figs. 3 and 4 evidence that the addition of the dye to the liquid crystalline matrix causes the reduction of the $x_{D}$ value at given reduced temperature. This means that the presence of the guest molecules in T15 


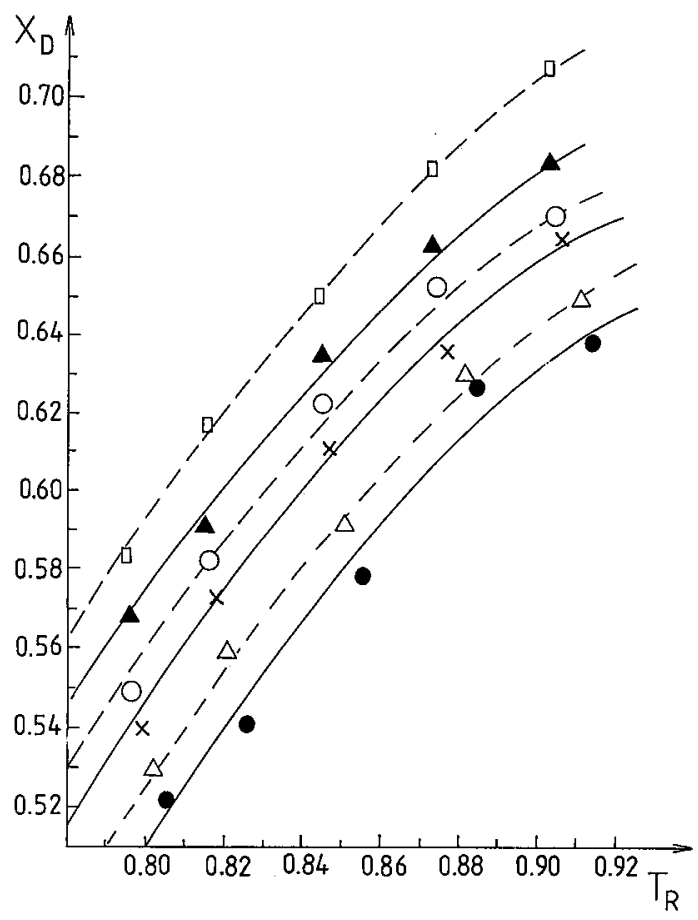

Fig. 3. Temperature dependence of the dimer fraction in the specimens investigated. The points are experimental results. Lines are visual guides. Dye concentration: $10^{-1} \mathrm{M}$ $\left.(\bullet), 6.6 \times 10^{-2} \mathrm{M}(\triangle), \mathrm{p}\right), 3.3 \times 10^{-2} \mathrm{M}(\times), 10^{-2} \mathrm{M}(\mathrm{o}), 3.3 \times 10^{-3} \mathrm{M}$ (fulltriangle); pure T15 (口) .

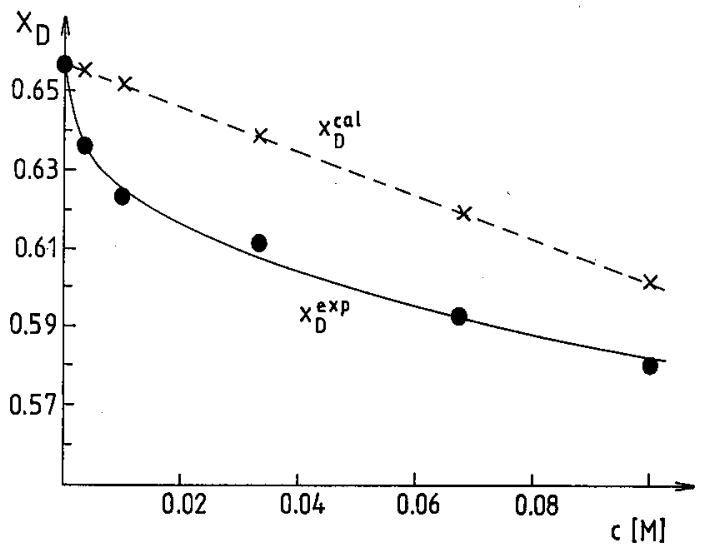

Fig. 4. Concentration variation of the dimer fraction in the dye-liquid crystal mixture at reduced temperature $T_{\mathrm{R}}=0.850$. 
perturbs the monomer-dimer equlibrium of the nematic host. The amount of the associated molecules in the guest-host mixture is strongly dependent on the dye concentration, but there is no linear relation between both (Fig. 4). The largest changes of the $x_{D}$ value occur at the low concentration of the dye. A similar effect has been observed previously by the studies of the dielectric properties of the guest-host systems in the isotropic phase $[12,27]$.

The dye molecule used in this paper has the strongly polar $-\mathrm{NO}_{2}$ group in the 4-position of the thiazolic sulphur atom. In the previous papers $[12,13,17$, 27] the changes in the monomer-dimer equilibrium in the nematics after addition of the dye with the $-\mathrm{NO}_{2}$ end group have been interpreted in terms of the strong interaction of this group with the polar -CN groups of the cyano-substituted liquid crystals. The new results confirm this assumption. However, the question appears in what way so small amount of the dye molecules (concentration of $10^{-1} \mathrm{M}$ means about 3 molecules of the guest per 100 molecules of T15) can affect so strongly the dipolar association of the host matrix?

The dashed line in Fig. 4 presents the values of $x_{\mathrm{D}}$ calculated by assuming that each dye molecule causes the dissociation of the one liquid crystalline dimer into the single molecules. Comparison of the calculated and experimental results indicates that short-range interactions in the guest-host mixtures are much more complicated than it would follow from assuming the occurence of the simple dissociation process only. It should be accepted that dipolar interaction among polar groups of the liquid crystal and dye molecules is so strong, that one dye molecule causes the dissociation of the more than one host dimer. The lack of the proportionality between the dimer fraction and the dye concentration could, however, indicate the possibility of the occurence of two competitive processes at higher guest concentrations: dissociation of the bimolecular associates of the liquid crystal into the single molecules with the simultaneous formation of the heteroassociates composed of one dye and one T15 molecules. The observed decrease of the $d_{\mathrm{D}}$ value with the increasing dye concentration can confirm this suggestion, because the length of the guest molecule, calculated from the standard bond lengths and angles [29] including van der Waals radii of the terminal atoms [30], is smaller than the length of T15 molecule, calculated in the same way [17].

Finally, it should be noticed that the molecular organization in the guest-host mixture is a result of the competitive interactions of various kind. It seems that interaction between permanent dipoles plays here the predominant role, but not the only one. The repulsive intermolecular interaction caused by packing or steric effects, dispersive attractive interaction and the anisotropy of them as well as the possibility of the formation of the aggregates larger than dimers should also be taken into account.

\section{Acknowledgements}

D.B. gratefully acknowledges an Alexander von Humboldt fellowship during her stay in Darmstadt. 


\section{References}

[1] M. Born, Sitz. d. Phys.-Math. 25, 614 (1916).

[2] J. van der Veen, W.H. de Jeu, A.H. Grobben, J. Boven, Mol. Cryst. Liq. Cryst. 17, 291 (1972).

[3] W. Maier, A. Saupe, Z.Naturforsch. A 13, 564 (1958); 14, 882 (1959); 15, 287 (1960); 16, 262 (1961).

[4] D.A. Dunmur, A.E. Tomes, Mol. Cryst. Liq. Cryst. 97, 241 (1983).

[5] W.H. de Jeu, Physical Properties of Liquid Crystalline Materials, ed. Gordon and Breach, Science Publishers, New York, 1980.

[6] ] W. Dannhauser, A.F. Fltckinger, J. Phys. Chem. 68, 1814 (1968).

[7] D. Lippens, J.P. Parneix, A. Chapoton, J.Phys. (Paris) 38, 1465 (1977).

[8] P.E. Cladis, D. Guillon, W.B. Daniels, A.C. Griffin, Mol. Cryst. Liq. Cryst. 56, 89 (1979).

[9] D.A. Dunmur, W.H. Miller, Mol. Cryst. Liq. Cryst. 60, 281 (1980).

[10] Hp. Schad, M.A. Osman, J. Chem. Phys. 75, 880 (1981).

[11] L.G.P. Dalmolen, S.J. Picken, A.F. de Jong, W.H. de Jeu, J. Phys. (Paris) 46, 1443 (1985).

[12] D. Bauman, W. Haase, Mol. Cryst. Liq. Cryst. 168, 155 (1989).

[13] D. Bauman, G. Czechowski, J. Jadzyn, Acta Phys. Pol. A75, 697 (1989).

[14] A.J. Leadbetter, R.M. Richardson, C.N. Colling, J. Phys. (Paris) 36, C1-37 (1975).

[15] G.J. Brownsey, A.J. Leadbetter, Phys. Rev. Lett. 44, 1608 (1980); J. Phys. Lett. (Paris) 42, L-135 (1980).

[16] W. Haase, Z.-X. Fan, H.J. Mtller, J. Chem. Phys. 89, 3317 (1988).

[17] D. Bauman, Z.-X. Fan, W. Haase, Liquid Crystals 6, 239 (1989).

[18] D. Bauman, Mol. Cryst. Liq. Cryst. 174, 1 (1989).

[19] W. Klamke, Z.-X. Fan, W. Haase, H.J. Mtller, M. Gallardo, Ber. Bunsenges. Phys. Chem. 93, 478 (1989).

[20] W. Haase, O. Trinquet, U. Quotschalla, J. Foitzik, Mol. Cryst. Liq. Cryst. 148, 15 (1987).

[21] D. Bauman, Mol. Cryst. Liq. Cryst. 159, 197 (1988).

[22] D. Bauman, Mol. Cryst. Liq. Cryst. 172, 41 (1989).

[23] D.E. Martire, in: The Molecular Physics of Liquid Crystals ed. by G.R. Luckhurst and G.W. Gray, Academic Press, London, New York, San Francisco, 1979, Chapter 10.

[24] F.C. Saunders, L. Wright, M.G. Clark, in: Liquid Crystals and Ordered Fluids, vol. 4, ed. by A.C. Griffin and J.F. Johnson, Plenum Press, New York, London, 1984.

[25] P. Diot, J.K. Foitzik, W. Haase, Rev. Phys. Appl. 20, 121 (1985).

[26] J. Cognard, T. Hieu Phan, Mol. Cryst. Liq. Cryst. 68, 207 (1989).

[27] D. Bauman, Acta Phys. Pol. A76, 641 (1989).

[28] A. de Vries, Mol. Cryst. Liq. Cryst. 131, 125 (1985).

[29] . Tables of the Interatomic Distances and Configuration in Molecules and Ions, published by The Chemical Society, Burlington House, London, 1958.

[30] A. Bondi, J. Phys. Chem. 68, 441 (1964). 